\title{
XMM-Newton observation of a distant X-ray selected cluster of galaxies at $z=1.26$ with possible cluster interaction
}

\author{
Yasuhiro Hashimoto ${ }^{1,2}$, Günther Hasinger ${ }^{1,2}$, Monique Arnaud ${ }^{3}$, Piero Rosati ${ }^{4}$, and Takamitsu Miyaji ${ }^{5}$ \\ 1 Astrophysikalisches Institut Potsdam, An der Sternwarte 16, 14482 Potsdam, Germany \\ 2 Max-Planck-Institut für extraterrestrische Physik, Giessenbachstrasse 85748 Garching, Germany \\ 3 Service d'Astrophysique CEA Saclay, 91191 Gif-sur-Yvette Cedex, France \\ 4 European Southern Observatory, 85748 Garching, Germany \\ 5 Department of Physics, Carnegie Mellon University, 5000 Forbes Ave., Pittsburgh, PA 15213, USA
}

Received 20 April 2001 / Accepted 29 October 2001

\begin{abstract}
We report on the XMM-Newton observation of RX J1053.7+5735, one of the most distant $(z=1.26)$ X-ray selected clusters of galaxies, which also shows an unusual double-lobed X-ray morphology, indicative of possible cluster-cluster interaction. The cluster was discovered during our ROSAT deep pointings in the direction of the Lockman Hole. The XMM-Newton observations were performed with the European Photon Imaging Camera (EPIC) during the performance verification phase. Total effective exposure time was $\sim 100 \mathrm{ksec}$. The best fit temperature based on a simultaneous fit of spectra from all EPIC cameras (pn+MOS) is $4.9_{-0.9}^{+1.5} \mathrm{keV}$. Metallicity is poorly constrained even using the joint fit of all spectra, with an upper limit on the iron abundance of 0.62 solar. Using the best fit model parameters, we derived an unabsorbed $(0.2-10) \mathrm{keV}$ flux of $f_{0.2-10}=3.0 \times$ $10^{-14} \mathrm{erg} \mathrm{cm}^{-2} \mathrm{~s}^{-1}$, corresponding to a bolometric luminosity of $L_{\mathrm{bol}}=3.4 \times 10^{44} h_{50}^{-2} \mathrm{erg} \mathrm{s}^{-1}$. Despite the fact that it was observed at fairly large off-axis angle, the temperature errors are much smaller compared with those of typical measurements based on ASCA or Beppo-Sax observations of high- $z(z>0.6)$ clusters, demonstrating the power of the XMM for determining the X-ray temperature for high- $z$ clusters. The measured temperature and luminosity show that one can easily reach the intrinsically X-ray faint and cool cluster regime comparable with those of $z \sim 0.4$ clusters observed by past satellites. The new cluster temperature and $L_{\mathrm{bol}}$ we have measured for RX J1053.7+5735 is consistent with a weak/no evolution of the $L_{\mathrm{bol}}-T_{\mathrm{x}}$ relation out to $z \sim 1.3$, which lends support to a low $\Omega_{\mathrm{m}}$ universe, although more data-points of $z>1$ clusters are required for a more definitive statement. The caution has to be also exercised in interpreting the result, because of the uncertainty associated with the dynamical status of this cluster.
\end{abstract}

Key words. galaxies: clusters: general - X-rays: galaxies - galaxies: evolution

\section{Introduction}

Clusters of galaxies can be identified to high redshift and can be used as tracers of the evolution of structure. Since the evolution of structure depends on parameters such as $\Omega_{\mathrm{m}}, \Lambda, \& P(k)$ in hierarchical cosmologies, the study of the evolution of galaxy clustering provides us with an important constraint on the cosmology (e.g. Press \& Schechter 1974; Peebles et al. 1989; Eke et al. 1996). $\mathrm{X}$-ray observations provide a powerful and unique means of selecting the clusters and characterizing their properties, as X-ray flux is proportional to the square of the electron density, and therefore less affected than optical data by the superposed structures. Measurements of the gas

Send offprint requests to: Y. Hashimoto,

e-mail: hashimot@aip.de temperatures in high redshift clusters of galaxies strongly constrain cosmological models because cluster temperatures are closely related to cluster masses, and the evolution of the cluster mass function with redshift is quite sensitive to cosmological parameters.

X-ray cluster surveys based on ROSAT-PSPC data (e.g. Rosati et al. 1995, 1998; Scharf et al. 1997; Burke et al. 1997; Vikhlinin et al. 1998) detect sizable samples of distant clusters $(z>0.5)$. Although the number of high- $z$ clusters has significantly increased, intracluster medium (ICM) properties (such as X-ray temperature: $T_{\mathrm{x}}$ ) at high redshift are still largely unexplored. This is due to the limited effective area and spatial resolution of the past X-ray missions; only long ASCA/Beppo-SAX observations, with a broad energy response, have allowed studies of the high- $z$ cluster ICM properties. Only a few high- $z$ 
$(z>0.6)$ clusters have directly measured X-ray temperatures (e.g. Donahue et al. 1999; Della Ceca et al. 2000). Moreover, these high- $z$ samples are inevitably biased toward intrinsically X-ray bright, and thus high temperature clusters, which complicates the investigation of ICM evolution.

Advent of Chandra, with its bigger effective area and higher angular resolution than the past satellites, made us possible to effectively study the ICM properties of high$z$ clusters. Chandra's high angular resolution, in particular, greatly helps us to avoid confusion with point sources (e.g. Stanford et al. 2001; Borgani et al. 2001; Jeltema et al. 2001; Cagnoni et al. 2001). Unfortunately, for those with directly measured $T_{\mathrm{x}}$, the errors on $T_{\mathrm{x}}$ are still relatively large. Moreover, Chandra is not particularly sensitive to low surface brightness emission, which is sometimes unfavorable for the analysis of faint X-ray features often present in high- $z$ clusters.

The XMM-Newton (XMM) pn and MOS CCD cameras have $\sim 10$ times larger effective area than ASCA GIS/SIS \& Beppo-SAX MECS (in addition to the fact that it has significantly sharper PSF than ASCA \& BeppoSax). Even compared with Chandra ACIS, the effective area is more than 5 times larger which helps to increase the accuracy of the $T_{\mathrm{x}}$ determination per given exposure time. With its high throughput and moderate (compared with Chandra) angular resolution, XMM is extremely sensitive to low surface brightness X-ray emission. These properties make XMM pn/MOS very suitable instruments to investigate the ICM properties of distant clusters.

In this paper, we report on the XMM observation of one of the most distant X-ray selected clusters $(z=1.26)$ of galaxies, which also shows an unusual double-lobed X-ray morphology. This cluster, RX J1053.7+5735, was discovered during our deep ROSAT HRI pointings in the direction of the "Lockman Hole", a line of sight with exceptionally low HI column density (Hasinger et al. 1998a). RX J1053.7+5735 is one of the most distant clusters ever selected by diffuse X-ray emission (for another most distant X-ray selected cluster at $z=1.26$, RX J0848.9+4452, see Rosati et al. 1999; Stanford et al. 2001). Together with the fact that its X-ray morphology is clearly double-lobed and highly anisotropic, which may well be a sign of a cluster in the making, the RX J1053.7+5735 may provide us with unique information to better understand the evolution of clusters and galaxies in the early universe. Throughout the paper, we use $H_{0}=100 h \mathrm{~km} \mathrm{~s}^{-1} \mathrm{Mpc}^{-1}$ and $q_{0}=0.5$.

\section{RX J1053.7+5735}

The X-ray source RX J1053.7+5735 was discovered during our deep pointings of $1.31 \mathrm{Msec}$ with ROSAT HRI in the direction of the Lockman Hole. Figure 1 shows the HRI contours superposed on the color image made from $V, R$, and $I$ (color coded in blue, green, and red) band exposures, where North is up and East is left. The HRI image revealed a clearly extended X-ray emission with

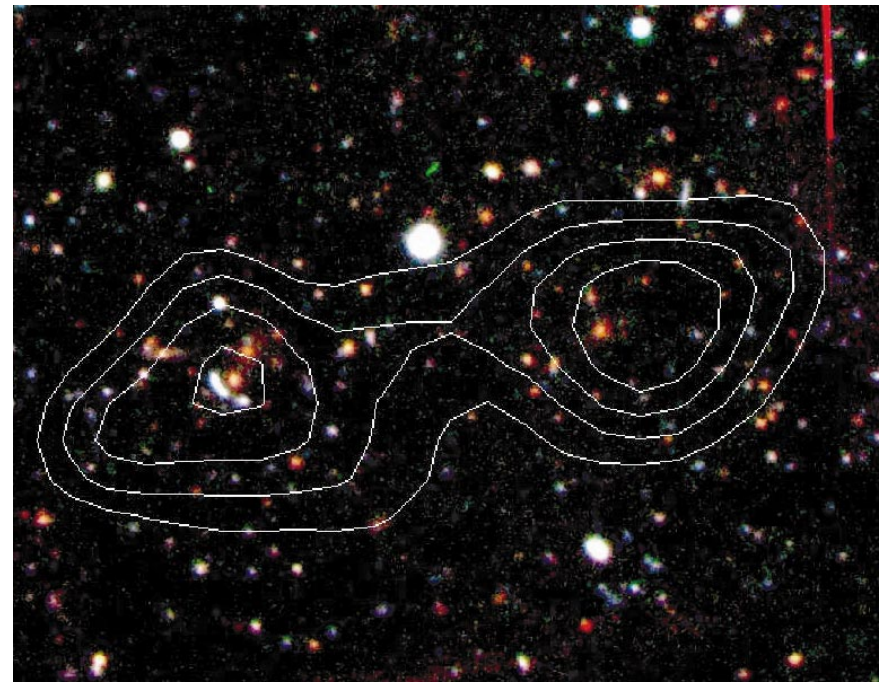

Fig. 1. The ROSAT HRI contours of the cluster RX J1053.7+5735 overlaid on the color image made from $V, R$, and $I$ (color coded in blue, green, and red) band exposures. North is up and East is left.

unusual double-lobed X-ray morphology. The angular size of the source is $1.7 \times 0.7 \operatorname{arcmin}^{2}$ and its X-ray flux is $2 \times$ $10^{-14} \mathrm{erg} \mathrm{cm}^{-2} \mathrm{~s}^{-1}$ (Hasinger et al. 1998b). Our subsequent deep optical/NIR imaging follow-ups $(V<26.5$, $R<25, I<25, K<20.5)$ with LRIS and NIRC on Keck, and the Calar Alto Omega Prime camera revealed a bright 7 arcsecond-long arc with an integrated magnitude of $R=21.4$, and an overdensity of galaxies in both X-ray lobes (e.g. Thompson et al. 2001). Further Keck LRIS/NIRSPEC spectroscopic observations on the bright arc and one of the brightest possible member galaxies confirmed that the bright arc is a lensed galaxy at a redshift $z=2.57$ and the galaxy is at a redshift of $z=1.263$ (Thompson et al. 2001). Deep VRIzJHK photometry data also produced concordant photometric redshifts for more than 10 objects at redshift of $z \sim 1.3$, confirming that at least the eastern lobe is a massive cluster at high redshift. The improbability of chance alignment and similarity of colors for the galaxies in the two X-ray lobes are consistent with the western lobe also being at $z \sim 1.3$ (see Thompson et al. 2001 for further details). The present data are all consistent with the identification of the X-ray source with one or two high redshift cluster(s) of galaxies. The spatial distribution of the X-ray gas and cluster galaxies in this system suggest that it could be a valuable example of a dynamically unsettled high redshift cluster, however, further optical, NIR, and X-ray investigations are planned for a more definitive statement about the dynamical state of this interesting cluster.

\section{XMM observations and data reduction}

The XMM observations of the cluster RX J1053.7+5735 were performed with the European Photon Imaging Camera (EPIC) during the period April 27-May 19, 2000 as a part of the performance verification (PV) phase 
Lockman Hole observation centered on the sky position RA 10:52:43, DEC +57:28:48 (J2000), for a total exposure time of $\sim 190 \mathrm{ksec}$. The cluster center is at an off-axis angle of $\sim 10^{\prime}$ from the pointing center, and this causes a vignetting effect, on the average, of a factor of about two with respect to the pointing center. The EPIC cameras were operated in the standard full-frame mode. The thin filter was used for the pn camera, while the thin and thick filters were used for the two MOS cameras. The pn detector consists of 12 CCDs each $13.6 \times 4.4$ arcmin, with energy resolutions of $6.7 \%$ at $1 \mathrm{keV}$, while MOS detectors consist of 7 CCDs each $10.9 \times 10.9$ arcmin with energy resolutions of $5.7 \%$ at $1 \mathrm{keV}$. The sensitive FOV of both detector is about $30^{\prime}$ in diameter and the energy range is both 0.1 to $15 \mathrm{keV}$. (For further details of the PV Lockman Hole observation, see Hasinger et al. 2001.)

Light curves were visually inspected, and time intervals with high background $(0.5-10 \mathrm{keV}$ count rate higher than $8 \mathrm{cts} / \mathrm{s}$ for the pn and $3 \mathrm{cts} / \mathrm{s}$ for MOS) were excluded. We used both single and double events. The remaining effective exposure times for each of three detectors were approximately $100 \mathrm{ksec}$. The astrometries calculated from the WCS keywords were offset by $5^{\prime \prime}-25^{\prime \prime}$ from the known optical counterparts. Thus, a transversal shift and a rotation angle were fit for each dataset, leading to residual systematic position errors of $1^{\prime \prime}-2^{\prime \prime}$.

Figure 2 shows the exposure corrected XMM image of RX J1053.7+5735 in the $0.5-2 \mathrm{keV}$ band, an energy range where the bulk of the redshifted cluster emission falls upon and therefore the contrast against the background is the strongest. The image was created by combining all events from three (pn, MOS1, and MOS2) cameras. The raw data were smoothed with a Gaussian with $\sigma=7^{\prime \prime}$. The lowest contour is $2 \sigma$ above the background, and the contour interval is $0.5 \sigma$. A combined exposure map was calculated for the pn plus MOS cameras.

Figure 2 clearly shows that the cluster emission is extended and double-lobed, consistent with the ROSAT HRI image, and its shape is indicative of non-regularity of this cluster. Unfortunately, the pointing variation between the different revolutions was not large enough to clearly remove the effect of inter-CCD gaps, which happen to run through the cluster, particularly around the eastern lobe, and this somewhat distorted the level and shape of the eastern-lobe contours.

\section{Spectral analysis}

The spectra were extracted from an elliptical region centered at the cluster image with a semi-major/minor axis 0.9 and 0.6 , respectively (corresponding to 0.23 and $0.16 \mathrm{Mpc} / h$ at $z=1.263)$. The backgrounds were estimated from a region of the detector at the same offaxis angle surrounding the cluster, after removing point sources. We obtain $791 \pm 37$ and $519 \pm 30$ net counts (in the $0.2-10.0 \mathrm{keV}$ band) for pn and $\operatorname{MOS}(1+2)$, respectively. We regrouped the counts in order to have a $S / N \geq 3$ in each bin after the background subtraction.

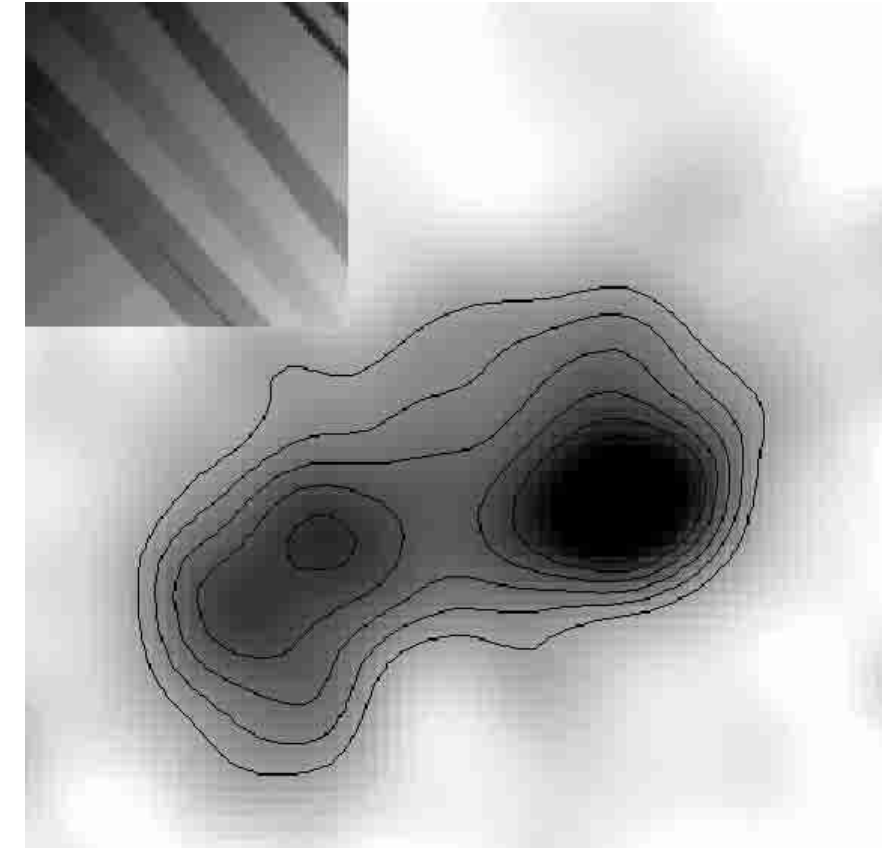

Fig. 2. The exposure corrected XMM image of the cluster RX J1053.7+5735 in the $0.5-2 \mathrm{keV}$ band. The image was created by combining all events from three (pn, MOS1, and MOS2) cameras. North is up and East is left. The raw data were smoothed with a Gaussian with $\sigma=7^{\prime \prime}$. The lowest contour is $2 \sigma$ above the background, and the contour interval is $0.5 \sigma$. Inset shows a combined ( $\mathrm{pn}+\mathrm{MOS})$ exposure map $(0.5-$ $2 \mathrm{keV}$ ) for the area identical to the cluster image. Inter-CCD gaps happen to run through the cluster, in particular through the eastern lobe, and this somewhat distorted the level and shape of the contours.

We fitted the spectra with a thermal plasma emission model from Raymond \& Smith (1977) using the XSPEC 11.0 with respective response matrices (Haberl 2001). For the MOS cameras, two filters were used alternately, thus we summed all the MOS spectra according to the used filter and fit two sets of spectra jointly. We fixed a characteristic column density of neutral hydrogen $N_{\mathrm{H}}$ at a value of $5.6 \times 10^{19} \mathrm{~cm}^{-2}$ and redshift at $z=1.263$. For the case of a joint fit, each spectrum was fitted with its own normalization, but with common temperature (and metallicity, if applicable).

In Fig. 3, and Table 1, the results of the best-fits are shown. Figure 4 shows the two-parameter $\chi^{2}$ contours

Table 1. Results of spectral fits for cluster RX J1053.7+5735.

\begin{tabular}{lrrr}
\hline \hline Detector & $\begin{array}{r}k T^{a} \\
(\mathrm{keV})\end{array}$ & $\begin{array}{r}\text { Abundance } \\
(\odot)\end{array}$ & $\chi^{2}$ (d.o.f.) \\
\hline pn & $4.8(3.7-6.3)$ & 0.3 (fixed) & $0.86(43)$ \\
MOS1+2 & $4.9(3.6-7.9)$ & 0.3 (fixed) & $0.78(29)$ \\
ALL & $4.9(4.0-6.4)$ & $<0.62$ & $0.83(72)$ \\
\hline
\end{tabular}

${ }^{a}$ The quoted temperature errors correspond to a single parameter error at $90 \%$ confidence. 

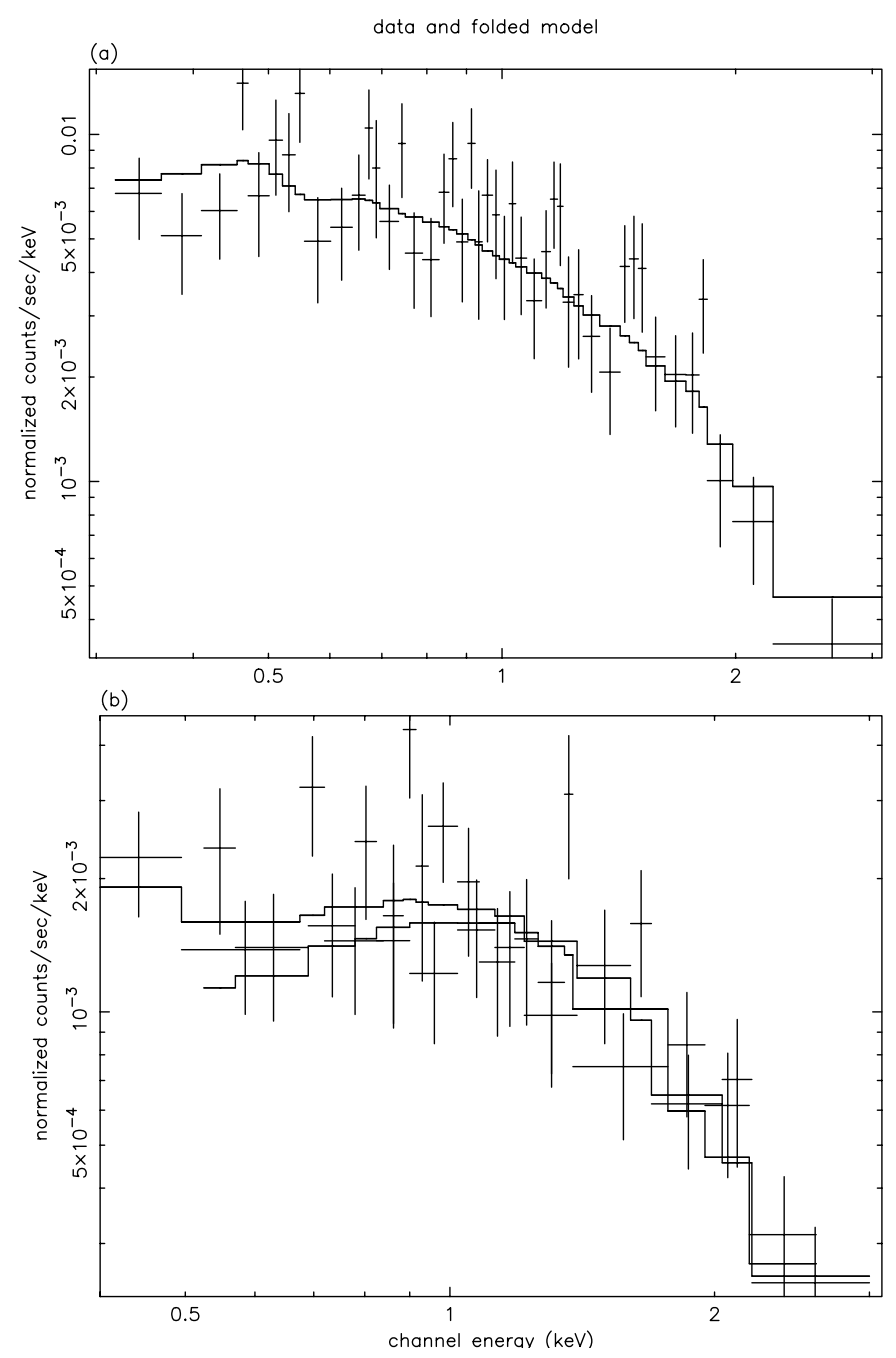

Fig. 3. Rebinned spectra and best-fit models for cluster RX J1053.7+5735 with pn a) and MOS1+2 b) cameras. The crosses are the observed spectrum and the solid line denotes best-fit model.

Table 2. Comparison between the two lobes (pn+MOS1+ MOS2).

\begin{tabular}{lrrll}
\hline \hline Lobe & $\begin{array}{r}k T \\
(\mathrm{keV})\end{array}$ & $\begin{array}{r}\text { Lbol } \\
\left(10^{44} \mathrm{erg} / \mathrm{s}\right)\end{array}$ & $\begin{array}{l}\text { Abundance } \\
(\odot)\end{array}$ & $\chi^{2}$ (d.o.f.) \\
\hline East & $3.7(2.7-4.9)$ & 1.5 & 0.3 (fixed) & $1.03(30)$ \\
West & $6.2(4.4-10.4)$ & 1.9 & 0.3 (fixed) & $0.82(43)$ \\
E+W & $4.9(4.0-6.4)$ & 3.4 & $<0.62$ & $0.83(72)$ \\
\hline
\end{tabular}

for the cluster metallicity and $T_{\mathrm{x}}$. The best fit temperature based on a simultaneous fit of all (pn+MOS) spectra is $4.9_{-0.9}^{+1.5} \mathrm{keV}$. All temperature uncertainties quoted are at the $90 \%$ confidence levels for a one-dimensional fit $\left(\Delta \chi^{2}=2.71\right)$. No prominent iron $\mathrm{K} \alpha$ complex is visible at $\sim 3 \mathrm{keV}$ (redshifted $6.7 \mathrm{keV}$ ). At $k T \sim 5 \mathrm{keV}$, and the cluster redshift, only the $\mathrm{K} \alpha$ is strong enough to constrain the abundance with our spectrum. As a result, metallicity is poorly constrained even using the joint fit of all spectra, with an upper limit on the single iron abundance

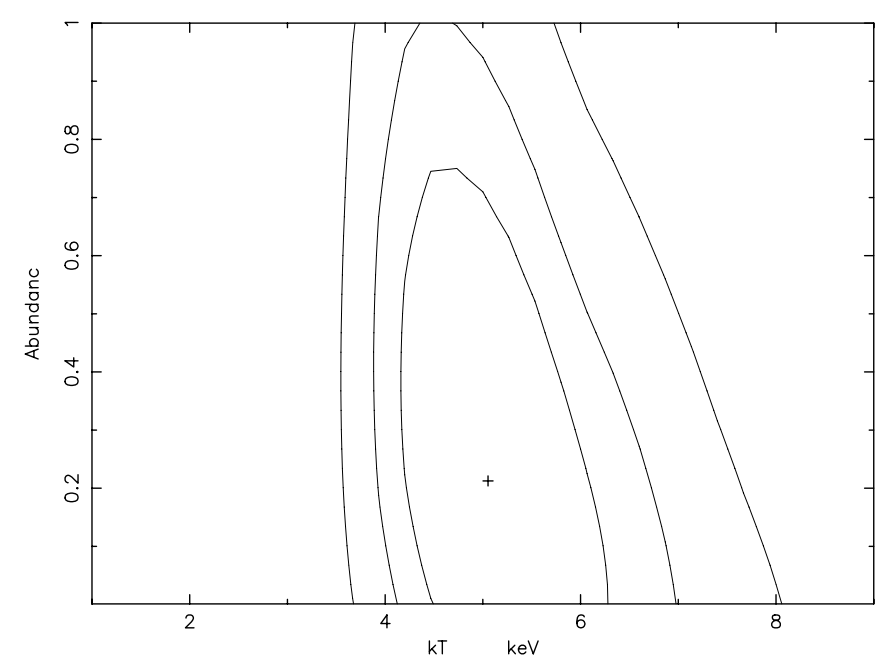

Fig. 4. Two-dimensional $\chi^{2}$ contours at $68.3 \%, 90 \%$, and $99 \%$ confidence levels $\left(\Delta \chi^{2}=2.30,4.61\right.$ and 9.21) for the cluster RX J1053.7+5735 temperature and abundance (in solar units) based on the joint fit for pn and MOS all together.

of 0.62 solar (with $68 \%$ confidence). Despite the fact that the cluster was observed at fairly large off-axis angle, the temperature errors are much smaller compared with those of typical measurements based on ASCA or Beppo-Sax observations of high- $z(z>0.6)$ clusters (Fig. 5a), and the errors are comparable with typically "on-axis" Chandra observations of $z \sim 0.8$ clusters (Fig. 5b), demonstrating the power of XMM for determining the X-ray temperature for high- $z$ clusters. Moreover, the measured temperature and luminosity show that one can easily reach the intrinsically X-ray faint and cool cluster regime comparable with those of $z \sim 0.4$ clusters observed by past satellites, which enables the investigation of the evolution of various cluster X-ray properties without additional evolutionary assumptions. Vignetting correction is estimated by using the average vignetting model between $0.5-7 \mathrm{keV}$, also averaged over the spectral extraction region. Using the best fit model parameters, we derived an unabsorbed $(0.2-10) \mathrm{keV}$ flux of $f_{0.2-10}=3.0 \times 10^{-14} \mathrm{erg} \mathrm{cm}^{-2} \mathrm{~s}^{-1}$, corresponding to a luminosity in the cluster rest frame of $L_{0.2-10}=3.1 \times 10^{44} h_{50}^{-2} \mathrm{erg} \mathrm{s}^{-1}$ and a bolometric luminosity of $L_{\mathrm{bol}}=3.4 \times 10^{44} h_{50}^{-2} \mathrm{erg} \mathrm{s}^{-1}$. Due to the still preliminary status of the current EPIC calibration, and some simplification about the vignetting correction, we estimate systematic flux errors on the order of $10 \%$.

Temperature and luminosity are also estimated for each (eastern and western) lobe of RX J1053.7+5735, using a circular (radius $=0.47$ ) extracting region centered at each lobe. Our fitting for each lobe using all data (pn+MOS) shows that $T_{\mathrm{x}}$ is $3.7_{-1.0}^{+1.2} \mathrm{keV}$ and $6.2_{-1.8}^{+4.2} \mathrm{keV}$, while $L_{\text {bol }}$ is $1.5 \times 10^{44} h_{50}^{-2} \mathrm{erg} \mathrm{s}^{-1}$ and $1.9 \times$ $10^{44} h_{50}^{-2} \mathrm{erg} \mathrm{s}^{-1}$, for eastern and western lobes, respectively (Table 2). For any other regions, such as the bridge between the two lobes, the counts are less than $10 \%$ of total cluster counts and not sufficient for any reasonable $T_{\mathrm{x}}$ investigations. 

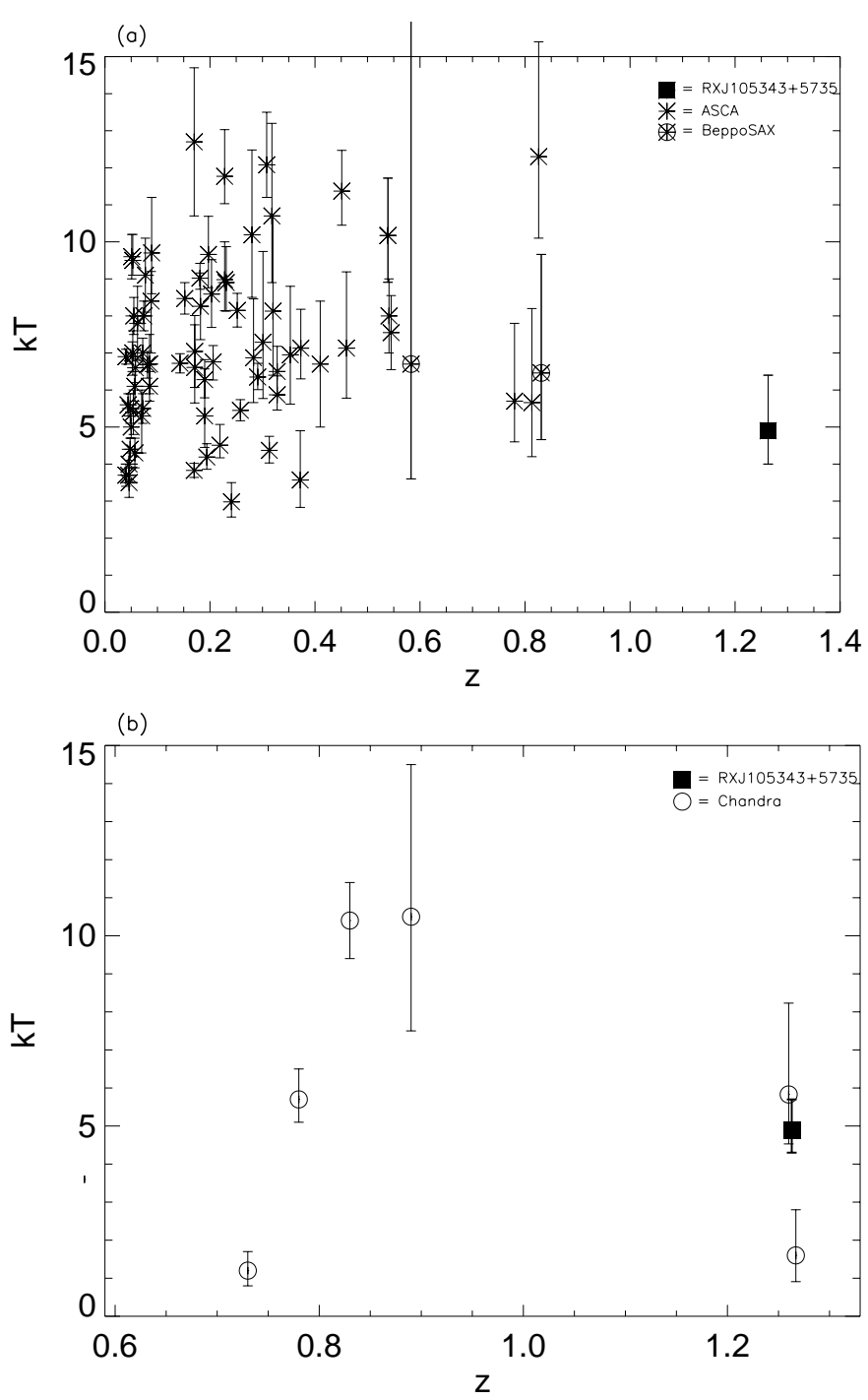

Fig. 5. a) New XMM temperature and its $2 \sigma$ error (90\%) for RX J1053.7+5735 plotted with other clusters observed by past satellites over various redshifts, taken from the literature (Markevitch 1998; Mushotzky \& Scharf 1997; Donahue et al. 1999; Della Ceca et al. 2000). Note that the temperature error for XMM measurement is comparable to those by ASCA for clusters at $z \sim 0.4$. Moreover, the measured temperature and luminosity showed that one can easily reach the intrinsically $\mathrm{X}$-ray faint and cool cluster regime comparable with those of $z \sim 0.4$ clusters observed by past satellites. b) Comparison between XMM temperature (RX J1053.7+5735) and new Chandra measurements of other high- $z$ clusters $(z>0.6)$ from Borgani et al. (2001). The error of RX J1053.7+5735 is changed to $1 \sigma(68.3 \%)$ to match quoted errors in Borgani et al. (2001).

\section{5. $L_{\mathrm{x}}-T_{\mathrm{x}}$ relation}

Clusters exhibit a correlation between their X-ray luminosity $\left(L_{\mathrm{x}}\right)$ and temperature $\left(T_{\mathrm{x}}\right)$. This correlation is often used to compare gas and dark matter contents in clusters, as $L_{\mathrm{x}}$ is connected with the gas while $T_{\mathrm{x}}$ is related to the total gravitating mass of the cluster. The cluster $L_{\mathrm{x}}-T_{\mathrm{x}}$ relationship therefore, can be used to examine the evolution of clusters and should be able to tell the range of

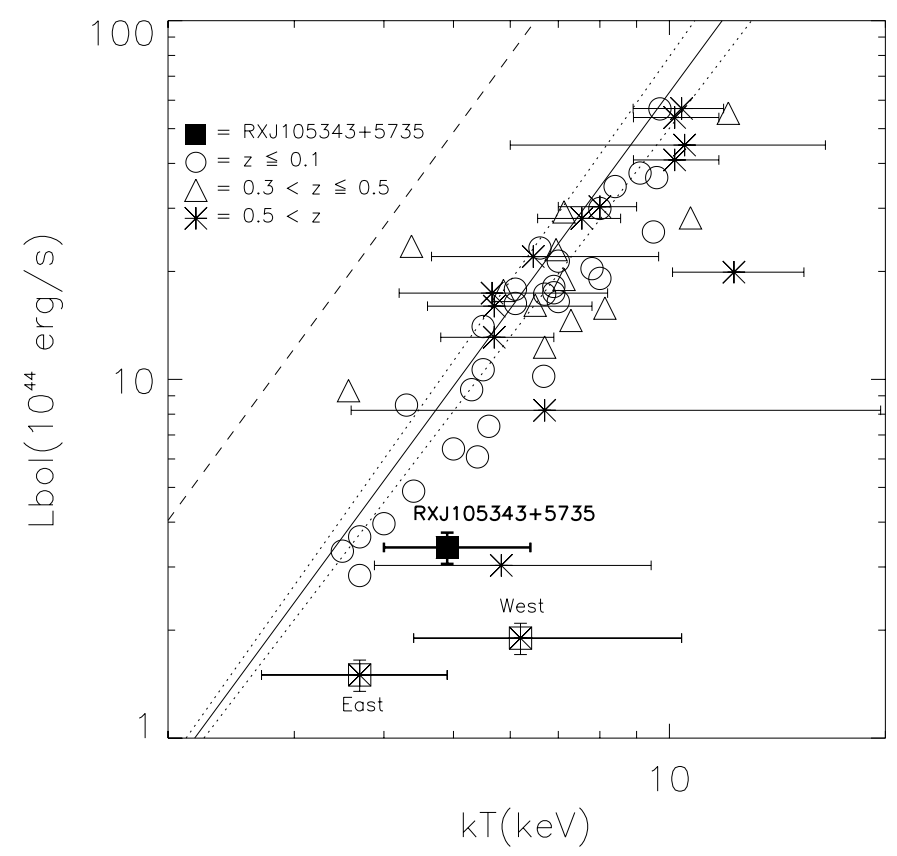

Fig. 6. Newly measured X-ray properties for a high- $z$ $(z=1.263)$ cluster, RX J1053.7+5735 (the square symbol), is compared with other clusters at various redshifts. The error on the temperature represents the $90 \%$ confidence range. The error on the RX J1053.7+5735 luminosity is $10 \%$. The eastern and western lobes of RX J1053.7+5735 (denoted as "East" and "West") are also shown. The solid line is the $L_{\mathrm{bol}}-T_{\mathrm{x}}$ relation of $L_{\mathrm{bol}}=10^{-0.92} T^{2.72}$ from Wu et al. (1999) together with $\pm 2 \sigma$ lines (dotted lines). The dashed line represents the evolving $L_{\mathrm{bol}}-T_{\mathrm{x}}$ relation in the form of $L_{\mathrm{bol}} \propto(1+z)^{2}$ at $z=1.263$, which required to make the observed non-evolving XLF to be consistent with a $\Omega_{\mathrm{m}}=1$ universe. The circles, triangle, \& stars denote low redshift $(z \leq 0.1)$, intermediate redshift $(0.3<z \leq 0.5)$, and high redshift $(0.5<z)$ clusters, respectively. For brevity, temperature error bars of only high- $z$ clusters are plotted. The new cluster temperature and $L_{\text {bol }}$ we have measured for RX J1053.7+5735 is consistent with a weak/no evolution of the $L_{\mathrm{bol}}-T_{\mathrm{x}}$ relation out to $z \sim 1.3$, which lends support to a low $\Omega_{\mathrm{m}}$ universe, although more data-points of $z>1$ clusters are required for a more definitive statement. The caution has to be also exercised in interpreting the result, because of the uncertainty associated with the dynamical status of this cluster.

redshifts where the cluster formation has taken place (e.g. Scharf \& Mushotzky 1997).

At low redshift $(z<0.1)$, the $L_{\mathrm{x}}-T_{\mathrm{x}}$ relation is well measured (e.g. Mushotzky 1984; Edge \& Stewart 1991; David et al. 1993) and expressed via power law: $L_{\mathrm{x}} \propto T_{\mathrm{x}}^{\alpha}$, with $\alpha \sim 2.6-2.9$ (Markevitch 1998; Allen \& Fabian 1998; Jones \& Forman 1999; Arnaud \& Evrard 1999).

At higher redshifts, however, no significant difference from the low redshift relations are reported, despite the fact that a relatively large evolution of the $L_{\mathrm{x}}-T_{\mathrm{x}}$ relation is required in order to account, within the frame work of the critical universe, for the weak evolution observed in the cluster X-ray luminosity function (XLF) out to $z \sim 0.8$ (Rosati et al. 1998). Mushotzky \& Scharf (1997) (see also Henry 2000), by comparing the clusters at $z<0.1$ with 
those at $z>0.1$, found no convincing evidence for a significant evolution of $L_{\mathrm{x}}-T_{\mathrm{x}}$ relationship out to $z \sim 0.4-0.5$, which is consistent with recent result of Wu et al. (1999) where they used the largest sample of clusters to date from the literature, and estimated $\alpha \sim 2.7$. For even higher redshifts, Donahue et al. (1999), using a complete sample of (five) high redshift $(z>0.5)$ EMSS clusters, have detected no significant evolution up to $z \sim 0.8$ (see also Della Ceca et al. 2000; Borgani et al. 2001).

In Fig. 6, we compare our newly measured X-ray properties for RX J1053.7+5735 with other clusters at various redshifts from the literature (Markevitch 1998; Mushotzky \& Scharf 1997; Donahue et al. 1999; Della Ceca et al. 2000; Borgani et al. 2001). The square denotes the new $L_{\mathrm{bol}}$ and $T_{\mathrm{x}}$ for RX J1053.7+5735. The error on the temperature represents the one dimensional $90 \%$ confidence range. The error bar on the RX J1053.7+5735 luminosity is $10 \%$. The eastern and western lobes of RX J1053.7+5735 (denoted as "East" and "West") are also separately shown. The solid line is the $L_{\mathrm{bol}}-T_{\mathrm{x}}$ relation of $L_{\mathrm{bol}}=10^{-0.92} T^{2.72}$ from Wu et al. (1999) together with $\pm 2 \sigma$ lines (dotted lines). The dashed line represents the evolving $L_{\mathrm{bol}}-T_{\mathrm{x}}$ relation in the form of $L_{\mathrm{bol}}$ $\propto(1+\mathrm{z})^{2}$ at $z=1.263$, which would be required to make the observed weak-evolving XLF to be consistent with a $\Omega_{\mathrm{m}}=1$ universe (Borgani et al. 1999). The circles, triangle, \& stars denote low redshift $(z \leq 0.1)$, intermediate redshift $(0.3<z \leq 0.5)$, and high redshift $(z>0.5)$ clusters, respectively. Total cluster luminosity may be somewhat underestimated because of the fundamental difficulty in measuring the luminosity of an extended source using a finite-sized extraction region. However, the treatments (if any) against the missing flux outside the extraction region often require difficult extrapolation and are not uniform for the low- $z$ cluster samples, or other high- $z$ samples in the literature. We therefore chose not to include the correction associated with the missing flux. Nevertheless, we crudely estimate the effect in our cluster using a profile from Neumann \& Arnaud (1999). We find that the uncertainty in $L_{\mathrm{x}}$ is on the order of $10 \%$, which is similar with the size of error bar shown in Fig. 6. The new cluster temperature and $L_{\text {bol }}$ we have measured for RX J1053.7+5735 is in agreement with other high- $z L_{\mathrm{x}^{-}}$ $T_{\mathrm{x}}$ analyses (e.g. Donahue et al. 1999; Della Ceca et al. 2000; Borgani et al. 2001).

\section{Discussion}

The dynamical state of high- $z$ clusters provides us with valuable and direct information on the formation and evolution of clusters. In hierarchical models, structures form from the bottom-up and thus, for many clusters, "formation" means an ongoing sequence of mergers and interactions with other clusters and groups. X-ray signatures of cluster interaction include surface brightness anomalies (e.g. non-symmetric, non-isotropic X-ray isophotes; substructures; elongate X-ray core; an X-ray peak offset from the peak of the galaxy distribution), and non-isothermal

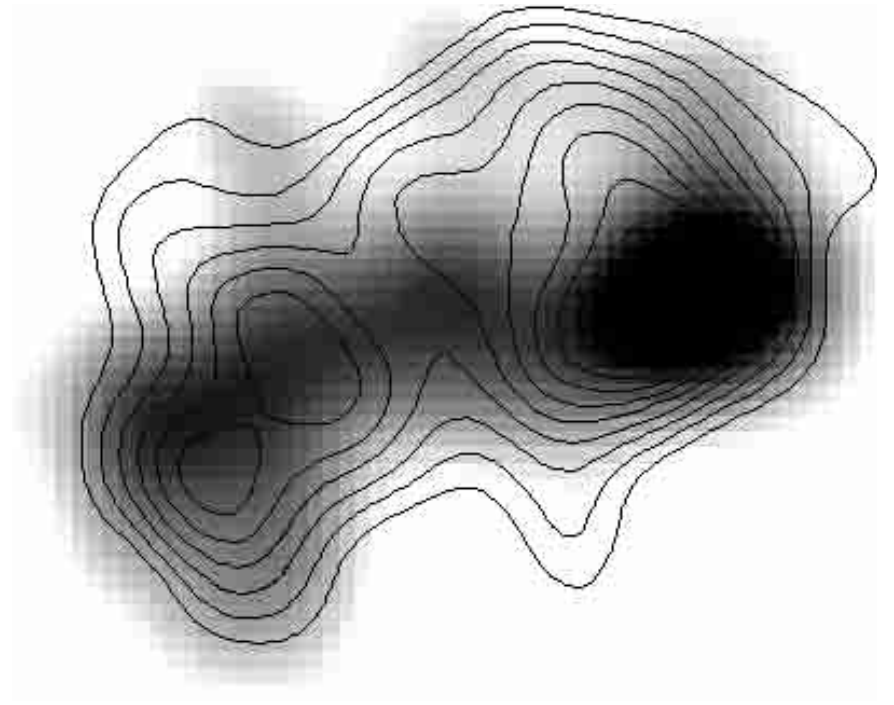

Fig. 7. The contour plot of $1-2 \mathrm{keV}$ image overlaid on the gray-scale $0.5-1 \mathrm{keV}$ image. Both images are smoothed with a Gaussian with $\sigma=7^{\prime \prime}$.

and asymmetric temperature distributions. Evidence for such cluster substructures and merger events in the local universe have been obtained with ROSAT and ASCA satellite (see Buote 2001 for a review). For higher redshifts $(z>0.5)$, the majority of clusters show clearly distorted X-ray morphology (e.g. Neumann \& Böhringer 1997; Gioia et al. 1999; Della Ceca et al. 2000; Neumann \& Arnaud 2000; Stanford et al. 2001; Jeltema et al. 2001), suggesting that they are in an unrelaxed state.

The fact that the X-ray morphology of RX J1053.7+5735 is double-lobed suggests that we may be seeing a merger event at $z \sim 1.3$, although our optical/NIR data are currently insufficient for a definitive statement about the dynamical state of this cluster. As for the $T_{\mathrm{x}}$ distribution, Fig. 7 shows the contour plot of an image in the $1-2 \mathrm{keV}$ band overlaid on the gray-scale image in the $0.5-1 \mathrm{keV}$ band. The figure shows some hint of a difference in the spatial distribution of the $1-2 \mathrm{keV}$ image contour with respect to the $0.5-1 \mathrm{keV}$ map, which may be interpreted as a $T_{\mathrm{x}}$ variation. The western lobe also shows a hint of higher $T_{\mathrm{x}}$, however, it is statistically inconclusive (Table 2). Future XMM (or Chandra) data of this cluster are needed, as well as more optical/NIR data, to improve our knowledge of the dynamical state of this cluster.

The new cluster temperature and $L_{\mathrm{x}}$ we have measured for RX J1053.7+5735 is consistent with a weak/no evolution of the $L_{\mathrm{x}}-T_{\mathrm{x}}$ relation out to $z \sim 1.3$, whereas a strong evolution of the $L_{\mathrm{x}}-T_{\mathrm{x}}$ relation is required to make the observed weak-evolving XLF to be consistent with a $\Omega_{\mathrm{m}}=1$ universe. Thus, our results could be interpreted as a support for a low $\Omega_{\mathrm{m}}$ universe, although more data-points of $z>1$ clusters are needed for a more definitive statement. The caution has to be also exercised in interpreting the result, because of the uncertainty associated with the dynamical state of the cluster. In fact, our results may 
emphasize the fundamental difficulty in constraining the cosmology from the evolution of the XLF and the $L_{\mathrm{x}}-T_{\mathrm{x}}$ relation, since the baseline of the comparison, the local $L_{\mathrm{x}}-T_{\mathrm{x}}$ relation and the theoretical $M-T$ relationships, are essentially valid for dynamically relaxed clusters (see also Ricker \& Sarazin 2001). If the majority of high- $z$ clusters are in the unrelaxed state, the impact of dynamically unrelaxed cluster states has to be carefully assessed. In particular, during mergers, clusters are expected to follow a complex track in the $L_{\mathrm{x}}-T_{\mathrm{x}}$ plane as shown in the numerical simulations (Ricker \& Sarazin 2001), and therefore, the relatively low luminosity of RX J1053.7+5735 in view of its temperature may be interpreted as further evidence for the unrelaxed state of the cluster.

Acknowledgements. We thank Pat Henry for a careful reading of the paper and useful comments. We also thank S. Majerowicz for his help in analyzing the MOS spectra. We acknowledge the referee's comments which improved the manuscript. Part of the work was supported by the Deutsches Zentrum für Luft- und Raumfahrt DLR project numbers 50 OX 9801 and 50 OR 9908.

\section{References}

Allen, S. W., \& Fabian, A. C. 1998, MNRAS, 297, L57

Arnaud, M., \& Evrard, A. E. 1999, MNRAS, 305, 631

Borgani, S., Rosati, P., Tozzi, P., \& Norman, C. 1999, ApJ, 517,40

Borgani, S., Rosati, P., Tozzi, P., et al. 2001 [astro-ph/0106428]

Buote, D. A. 2001, in Merging Processes in Clusters of Galaxies, ed. L. Feretti, I. M. Gioia, \& G. Giovannini (Dordrecht: Kluwer), in press

Burke, D. J., Collins, C. A., Sharples, R. M., et al. 1997, ApJ, 488, L83

Cagnoni, I., Elvis, M., Kim, D.-W., et al. 2001, ApJ, in press [astro-ph/0106066]

David, L. P., Slyz, A., Jones, C., et al. 1993, ApJ, 412, 479

Della Ceca, R., Scaramella, R., Gioia, I. M., et al. 2000, A\&A, 353,498

Donahue, M., Voit, G. M., Scharf, C. A., et al. 1999, ApJ, 527, 525
Edge, A. C., \& Stewart, G. C. 1991, MNRAS, 252, 428

Edge, A. C., Stewart, G. C., \& Fabian, A. C. 1992, MNRAS, 255,431

Eke, V. R., Cole, S., \& Frenk, C. S. 1996, MNRAS, 282, 263

Gioia, I. M., Henry, J. P., Mullis, C. R., Ebeling, H., \& Wolter, A. 1999 ApJ, 117, 2608

Haberl, F. 2001, private communication

Hasinger, G., Burg, R., Giacconi, R., et al. 1998a, A\&A, 329, 482

Hasinger, G., Giacconi, R., Gunn, J. E., et al. 1998b, A\&A, 340, L27

Hasinger, G., Altieri, B., Arnaud, M., et al. 2001, A\&A, 365, L45

Henry, J. P. 2000, ApJ, 534, 565

Jeltema, T. E., Canizares, C. R., Bautz, M. W., et al. 2001, ApJ, in press

Jones, C., \& Forman, W. 1999, ApJ, 511, 65

Markevitch, M. 1998, ApJ, 504, 27

Mushotzky, R. 1984, Phys. Scr., T7, 157

Mushotzky, R., \& Scharf, C. 1997, ApJ, 482, L13

Neumann, D. M., \& Böhringer, H. 1997, MNRAS, 289, 123

Neumann, D. M., \& Arnaud, M. 1999, A\&A, 348, 711

Neumann, D. M., \& Arnaud, M. 2000, ApJ, 542, 35

Peebles, P. J. E., Daly, R., \& Juskeiewiez, R. 1989, ApJ, 347, 563

Press, W., \& Schechter, P. 1974, ApJ, 187, 425

Raymond, J. C., \& Smith, B. W. 1977, ApJS, 35, 419

Ricker, P. M., \& Sarazin, C. L. 2001, ApJ, in press

Rosati, P., Della Ceca, R., Burg, R., Norman, C., \& Giacconi, R. 1995, ApJ, 445, L11

Rosati, P., Della Ceca, R., Norman, C., \& Giacconi, R. 1998, ApJ, 492, L21

Rosati, P., Stanford, S. A., Eisenhardt, P. R., et al. 1999, AJ, 118,76

Scharf, C. A., \& Mushotzky, R. 1997, ApJ, 485, L65

Scharf, C. A., Jones, L. R., Ebeling, H., et al. 1997, ApJ, 477, 79

Stanford, S. A., Holden, B., Rosati, P., et al. 2001, ApJ, in press [astro-ph/0012250]

Thompson, D., Pozzetti, L., Hasinger, G., et al. 2001, A\&A, 377, 778

Vikhlinin, A., McNamara, B. R., Forman, W., et al. 1998, ApJ, 502, 598

Wu, X.-P., Xue, Y.-J., \& Fang, L.-Z. 1999, ApJ, 524, 22 